\title{
Review: antidepressants plus benzodiazepines lead to fewer drop outs and less depression severity in major depression
}

\author{
Furukawa T, Streiner DL, Young LT. Antidepressant plus benzodiazepine for major depression. Cochrane Database Syst Rev \\ 2000;(4):CD001026 (latest version 29 Aug 2000). \\ Furukawa TA, Streiner DL, Young LT. Is antidepressant-benzodiazepine combination therapy clinically more useful? A \\ meta-analytic study.J Affect Disord 2001 Jul;65:173-7.

\section{QUESTION: In adults with major depression, does combination treatment with antidepressants and benzodiazepines lead to short term (< $8 \mathrm{wk})$ or long term $(>2 \mathrm{mo})$ symptomatic recovery or side effects?}

Source of funding: Ministry of Health and Welfare, Japan and Uehara Memorial Foundation, Japan.

For correspondence: Professor TA Furukawa, Department of Psychiatry, Nagoya City University Medical School, Mizuho-cho Mizuho-ku, Nagoya 467-8601, Japan. Fax +8528520837 .

Abstract and commentary also appear in

Evidence-Based Mental Health.

\section{Data sources}

Studies were identified by searching Medline, EMBASE/ Excerpta Medica, International Pharmaceutical Abstracts, Biological Abstracts, LILACS, PsycLIT, the Cochrane Library, and the trial register of the Cochrane Depression, Anxiety, and Neurosis Group (January 1972 to December 1998); hand searching major mental health and general medicine journals; scanning the

\section{COMMENTARY}

The valid review by Furukawa $e t$ al shows that benzodiazepines may add to the efficacy of antidepressants. The effect is strongest after 1 week, lasts until 4 weeks, and disappears after 6 to 8 weeks, although this last finding is based on data concerning only 162 patients.

What could cause this somewhat unexpected benefit? First, it may be that benzodiazepines prevent the occurrence of anxiety-related adverse effects in the first weeks of treatment, which may have led to fewer drop outs, particularly in the studies with selective serotonin reuptake inhibitors (SSRIs). The 2 studies (126 patients) evaluating SSRIs do not seem to exclude such a trend, but no subgroup analysis was done. However, the effect is still statistically significant after exclusion of drop outs.

Second, the beneficial effect may be because of a reduction in anxiety and sleep disturbance, leaving the core symptoms of low mood and anhedonia undisturbed. Such a subgroup analysis was not possible and would require collection of individual patient data. However, reduction in anxiety could also speed up or promote further remission.

Finally, a true additional effect of benzodiazepines would align with the increasing evidence for low $\gamma$-aminobutyric acid function in depression. ${ }^{1}$ The possible earlier onset of action is intriguing in this respect because pharmaceutical companies have been looking for this quality in new drugs without much success.

What are the clinical consequences? Clinicians are advised to prescribe benzodiazepines only to patients with severe anxiety and for no longer than 2 to 4 weeks. This review offers support for the relatively common practice of combining antidepressants with benzodiazepines for depressed patients who have severe symptoms of anxiety or who develop these while receiving antidepressants. Benzodiazepines should preferably be prescribed during the first weeks only and be withdrawn gradually. The only 2 studies in this review that investigated the risk for relapse after withdrawal reported numbers needed to harm of 2 and 7 .

$$
\begin{array}{r}
\text { Harm Gijsman, MD } \\
\text { University of Oxford, Oxford, UK }
\end{array}
$$

1 Shiah IS, Yatham LN. GABA function in mood disorders: an update and critical review. Life Sci 1998;63:1289-303.

\begin{tabular}{|c|c|c|c|c|}
\hline \multirow[b]{2}{*}{ Outcomes } & \multicolumn{2}{|c|}{ Weighted event rates } & \multirow[b]{2}{*}{ RRR $(95 \%$ Cl) } & \multirow[b]{2}{*}{ NNT (CI) } \\
\hline & Combined & Antidepressant alone & & \\
\hline Dropped out & $22 \%$ & $33 \%$ & $37 \%$ (19 to 51$)$ & 10 (6 to 22$)$ \\
\hline \multirow[t]{2}{*}{ Dropped out because of side effects } & $7 \%$ & $14 \%$ & $48 \%$ (14 to 68$)$ & $15(10$ to 40$)$ \\
\hline & & & RBI (CI) & NNT (Cl) \\
\hline$>50 \%$ reduction in depression at 4 weeks & $52 \%$ & $37 \%$ & $38 \%$ (15 to 66$)$ & $7(5$ to 15$)$ \\
\hline
\end{tabular}

Combined antidepressant and benzodiazepine treatment $v$ antidepressants alone for major depression*

${ }^{*}$ Abbreviations defined in glossary; RRR, RBI, NNT, and $\mathrm{Cl}$ calculated from data in article.

reference lists of identified articles; checking SciSearch; and making personal contacts.

\section{Study selection}

Studies were selected if they were randomised controlled trials comparing combined antidepressantbenzodiazepine treatment with antidepressants alone in adults with major depression. Studies were excluded if the antidepressant dose was $<100 \mathrm{mg}$ of imipramine, or its equivalent daily, or the duration of the trial was $<4$ weeks.

\section{Data extraction}

Data were extracted on patient characteristics, treatment type and dose, duration of follow up, and main outcomes.

\section{Main results}

9 studies (679 patients) met the selection criteria. The antidepressants studied were imipramine, desipramine, amitriptyline, maprotiline, nortriptyline, clomipramine, fluoxetine, and mianserin; benzodiazepines studied included triazolam, alprazolam, diazepam, chlordiazepoxide, flunitrazepam, lormetazepam, bentazepam, clonazepam, and mexazolam. Patients allocated to the combined treatment group were less likely to drop out of the study than were those in the antidepressant alone group (table). Patients in the combined treatment group were also less likely to drop out because of side effects (table). More patients in the combined treatment group showed a $>50 \%$ reduction from their baseline depression severity at 4 weeks than did those in the antidepressant alone group (worst case scenario for drop outs; table). This difference was no longer statistically significant at 8 weeks.

\section{Conclusion}

In adults with major depression, a combination of antidepressant and benzodiazepine treatment leads to fewer drop outs and less depression severity at 4 weeks than do antidepressants alone. 